\title{
Thick, Adherent Diamond Films on AIN with Low Thermal Barrier Resistance
}

Soumen Mandal, ${ }^{* \dagger \odot}$ Chao Yuan, ${ }^{\S}$ Fabien Massabuau, ${ }^{\| \odot}$ James W. Pomeroy, ${ }^{\S}$ Jerome Cuenca, ${ }^{\dagger}($ ) Henry Bland, ${ }^{\dagger}$ Evan Thomas, ${ }^{\dagger}$ David Wallis, ${ }^{\neq, \|}$Tim Batten, ${ }^{\perp}{ }^{\circ}$ David Morgan, ${ }^{\#}{ }^{\circ}$ Rachel Oliver, ${ }^{\|}$ Martin Kuball, ${ }^{*} \S$ and Oliver A. Williams ${ }^{*} \dagger$

${ }^{\dagger}$ School of Physics and Astronomy, and ${ }^{\ddagger}$ School of Engineering, Cardiff University, Cardiff CF24 3AA, U.K.

${ }^{\S}$ Center for Device Thermography and Reliability, University of Bristol, Bristol BS8 1TL, U.K.

"Department of Materials Science \& Metallurgy, University of Cambridge, Cambridge CB3 OFS, U.K.

${ }^{\perp}$ Renishaw plc., Wotton-under-Edge GL12 7DW, U.K.

${ }^{\#}$ Cardiff Catalysis Institute, School of Chemistry, Cardiff University, Cardiff CF10 3AT, U.K.

Supporting Information

ABSTRACT: The growth of $>100-\mu \mathrm{m}$-thick diamond layers adherent on aluminum nitride with low thermal boundary resistance between diamond and AlN is presented in this work. The thermal barrier resistance was found to be in the range of $16 \mathrm{~m}^{2} \cdot \mathrm{K} / \mathrm{GW}$, which is a large improvement on the current state-of-the-art. While thick films failed to adhere on untreated AlN films, AlN films treated with hydrogen/ nitrogen plasma retained the thick diamond layers. Clear differences in $\zeta$-potential measurement confirm surface modification due to hydrogen/nitrogen plasma treatment. An increase in non-diamond carbon in the initial layers of

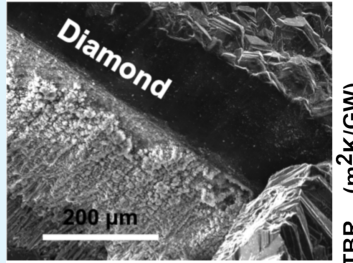

Thick diamond on ALN with lowest thermal boundary resistance of $16 \mathrm{~m}^{2} \mathrm{~K} / \mathrm{GW}$.

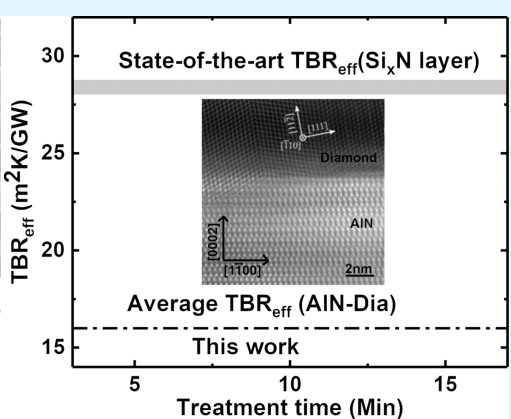
diamond grown on pretreated AlN is seen by Raman spectroscopy. The presence of non-diamond carbon has minimal effect on the thermal barrier resistance. The surfaces studied with X-ray photoelectron spectroscopy revealed a clear distinction between pretreated and untreated samples. The surface aluminum goes from a nitrogen-rich environment to an oxygen-rich environment after pretreatment. A clean interface between diamond and AlN is seen by cross-sectional transmission electron microscopy.

KEYWORDS: diamond, aluminum nitride, thermal barrier resistance, diamond seeding, diamond growth

\section{INTRODUCTION}

High electron mobility transistors (HEMTs) made from gallium nitride $(\mathrm{GaN})$ are attractive for high-power and high-frequency applications. ${ }^{1,2}$ Still, the full potential of GaN HEMT devices is yet to be realized due to less than effective thermal management. It has been shown that slight changes to the operating temperature of these devices can lead to a significant reduction in device lifetime. ${ }^{3,4}$ Current standards for effective thermal management in $\mathrm{GaN}$ devices entails the structures to be made from $\mathrm{GaN}$ grown on silicon carbide $(\mathrm{SiC}){ }^{5,6}$ Though $\mathrm{SiC}$ has a thermal conductivity of $\kappa_{\mathrm{SiC}} \approx$ $360-490 \mathrm{~W} / \mathrm{m} \cdot \mathrm{K},{ }^{7}$ resulting in good device performances, further improvement can be made if the $\mathrm{SiC}$ layer is replaced with diamond substrates $\left[\kappa_{\mathrm{Dia}} \approx 2100 \mathrm{~W} / \mathrm{m} \cdot \mathrm{K}^{8}\right.$ for single crystal and $\approx 1200 \mathrm{~W} / \mathrm{m} \cdot \mathrm{K}^{9-11}$ for a $100-\mu \mathrm{m}$-thick polycrystalline diamond layer (averaged through the nucleation layer to growth surface)]. Another approach can be to use large-area heteroepitaxial diamond films which can be grown by chemical vapor deposition on iridium substrates. ${ }^{12,13}$ But since they cannot be directly grown on AlN, only a wafer-bonding route may be suitable for such films. For cost-effective, direct-growth wafer scale implementation of diamond as a thermal management layer, polycrystalline films need to be used. This is due to the fact that it is possible to grow large-area wafer-scale polycrystalline diamond films, which is not the case for single crystals. Furthermore, it has been shown that the average through-plane thermal conductivity of $100-\mu \mathrm{m}$-thick polycrystalline diamond films exceeds the thermal conductivity of $\mathrm{SiC}$ by $>3$ times, which is required for a step-change in $\mathrm{GaN}$ device thermal management. ${ }^{14}$

For fabrication of $\mathrm{GaN}$-diamond devices, the diamond can be grown on $\mathrm{GaN}$, or $\mathrm{GaN}$ can be grown on diamond. The growth of $\mathrm{GaN}$ on diamond is possible only on single-crystal diamond and has led to promising devices. ${ }^{15,16}$ But, the high cost and small size of single-crystal diamond make them unattractive. In the past, polycrystalline diamond has been

Received: August 4, 2019

Accepted: October 11, 2019

Published: October 11, 2019 
grown on $\mathrm{GaN}$ with buffer layers, ${ }^{11,17}$ but the low thermal conductivity of the amorphous $\mathrm{SiN}$ adhesion layer is known to be a thermal bottleneck. ${ }^{18}$ Removing this thermal bottleneck between $\mathrm{GaN}$ and diamond will enable the full heat extraction potential of $\mathrm{GaN}$-on-diamond to be realized. Alternatively, the growth of thin diamond layers directly on GaN surface ${ }^{19}$ has been shown. However, it is not possible to grow a thick diamond layer $(>10 \mu \mathrm{m})$ on $\mathrm{GaN}$ due to the absence of any carbide bond between $\mathrm{GaN}$ and diamond layers and large differences in their thermal expansion coefficients. It has also been shown that $\mathrm{GaN}$ grown on sapphire is unsuitable for growth of thick diamond layers due to the thermal mismatch between diamond and sapphire. ${ }^{20}$ However, diamond can be grown on $\mathrm{AlN}^{21}$ This offers the possibility for a much improved GaN-on-diamond material structure. An AlN interlayer could be incorporated into a $\mathrm{GaN}$ buffer stack, grown onto, for example, a Si substrate. The Si substrate along with the AlGaN-based strain-relieving layers, needed for the growth of $\mathrm{GaN}$ onto $\mathrm{Si}$, can be removed and a selective etch can be used to expose the embedded AlN interlayer. The thick diamond substrate layer can be grown onto the back of the AlGaN/GaN device layers for optimal heat sinking. ${ }^{22}$ We note that there is also an AlN layer just at the interface between the Si substrate and GaN, though its utilization would not be useful in this context due to the high thermal resistance of the AlGaN strain-relieving layer as was previously demonstrated. ${ }^{23}$

In this work, the growth of a thick $(>100 \mu \mathrm{m})$ diamond layer on 250-nm-thick AlN layers has been successfully demonstrated and a possible model of its growth developed. The thermal barrier resistance of the interface was also measured and found to be much improved compared to state-of-the-art $\mathrm{GaN}$-on-diamond material. In the past, there have been attempts to grow thin diamond layers on $\mathrm{AlN}^{24-28}$ but the growth of $>50-\mu \mathrm{m}$-thick layers has not been demonstrated. The growth of diamond on an AlN surface by microwave plasma chemical vapor deposition also exposes the surface to hydrogen and methane plasma. Pobedinskas et al. ${ }^{29}$ had studied the effects of hydrogen and methane plasmas on sputtered AlN. They found that the films can be etched when exposed to plasma for long periods of time. Once the diamond film is coalesced, the AlN layer should be protected by the diamond layer. Cervenka et al. ${ }^{30}$ had studied the effect of hydrogen plasma on the nucleation density and surface morphology of diamond grown on single-crystal AlN. Similarly, Pobedinskas et al. ${ }^{31}$ described a method to enhance the seeding density on AlN. In the literature, there are other examples of plasma pretreatment on various other substrates for nucleation modification; in most cases, it has been done to enhance the nucleation density. ${ }^{32-37}$ The growth of thick layers on as-received AlN was not possible initially when untreated as-grown AlN surfaces were used, but after surface treatment (hydrogen/nitrogen plasma exposure before seeding) it was possible to grow thick layers on AlN thin films. Here as-received films refer to films that were not pretreated prior to diamond seeding. The $\zeta$-potential of the AlN surfaces before and after treatment has been measured. X-ray photoelectron spectroscopy was done to analyze the treated and untreated surfaces. Atomic force microscopy (AFM) was used to observe the diamond seeds on the surface of the substrates. The Raman maps have been taken of thin diamond films grown on as-received and pretreated AlN to determine the interface composition. The interface was imaged and electron energy loss spectroscopy was done using scanning transmission electron microscopy (STEM). Finally, the thermal barrier resistance $\left(\mathrm{TBR}_{\mathrm{eff}}\right)$ was measured using transient thermoreflectance measurements.

\section{RESULTS AND DISCUSSIONS}

2.1. $\zeta$-Potential of AIN Thin Films. The AlN layer used in this study was grown on $150 \mathrm{~mm}$ Si substrates in an Aixtron close-coupled showhead metal-organic chemical vapor deposition (MOCVD) system. Trimethylaluminum (TMAl) was used as the $\mathrm{Al}$ source and ammonia $\left(\mathrm{NH}_{3}\right)$ was used as the nitrogen source in hydrogen carrier gas. The single-crystal AlN film is grown with (0001) orientation and has an Al-polar growth surface. An indication of the crystal quality of the layer is given by the X-ray fwhm of the (0002) and (1011) peaks, which have values of 3300 and 5500 arcsec, respectively. The high X-ray fwhm values of the AlN are dominated by the large in-plane lattice mismatch between the AlN and Si substrate, which requires a high density of misfit dislocations at the interface. The $\zeta$-potential measurements were made in order to determine the charge of the AlN surface in water as a function of $\mathrm{pH}$. This is important, as it allows the determination of the most effective seeding solution, i.e., the necessary charge on the diamond nanoparticles in order to drive electrostatic attraction. The results of the $\zeta$-potential measurements are shown in Figure 1 . The $\zeta$-potentials as a function of electrolyte

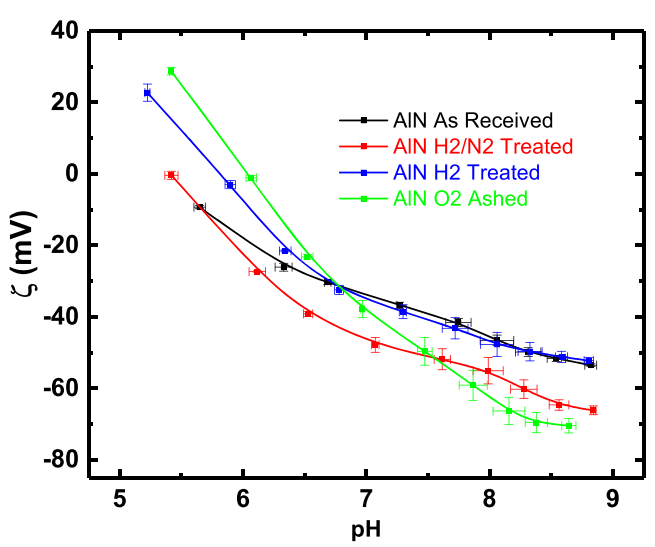

Figure 1. $\zeta$-Potential vs $\mathrm{pH}$ for as-received $\mathrm{AlN}$ and the same sample after pretreatment with $\mathrm{H}_{2} / \mathrm{N}_{2}$ plasma for $10 \mathrm{~min}$. A clear increase in negative $\zeta$-potential can be seen after treatment, indicating an increase in oxygen content on the surface. The $\zeta$-potentials of the AlN surface after $\mathrm{H}_{2}$ plasma treatment and oxygen ashing are also presented. The changes in $\zeta$-potential for these treatments are not as large as for $\mathrm{H}_{2} /$ $\mathrm{N}_{2}$ plasma at $\mathrm{pH} 7$.

$\mathrm{pH}$ of the as-received AlN substrates and AlN substrates treated with $\mathrm{H}_{2} / \mathrm{N}_{2}$ plasma, $\mathrm{H}_{2}$ plasma, and $\mathrm{O}_{2}$ plasma were measured. $\mathrm{H}_{2} / \mathrm{N}_{2}$ plasma pretreatment was needed for successful growth of a thick diamond layer on AlN. For comparison, the $\zeta$-potentials of $\mathrm{H}_{2}$-plasma- and $\mathrm{O}_{2}$-plasmatreated AlN surfaces were also measured. The main point of interest for seeding is the $\zeta$-potential in the $\mathrm{pH}$ range of $6-7$. This is the $\mathrm{pH}$ range of the diamond solution. In this range, the $\zeta$-potentials for samples treated with $\mathrm{H}_{2}$ plasma and $\mathrm{O}_{2}$ plasma remain close to that of the as-received substrates, staying in the range from -30 to $-35 \mathrm{mV}$. In contrast, the $\zeta$-potential of $\mathrm{H}_{2}$ / $\mathrm{N}_{2}$-plasma-treated substrates is enhanced and it is between -40 and $-45 \mathrm{mV}$ in the $\mathrm{pH}$ range of interest. Since an observable change in $\zeta$-potential was only seen in the case of 

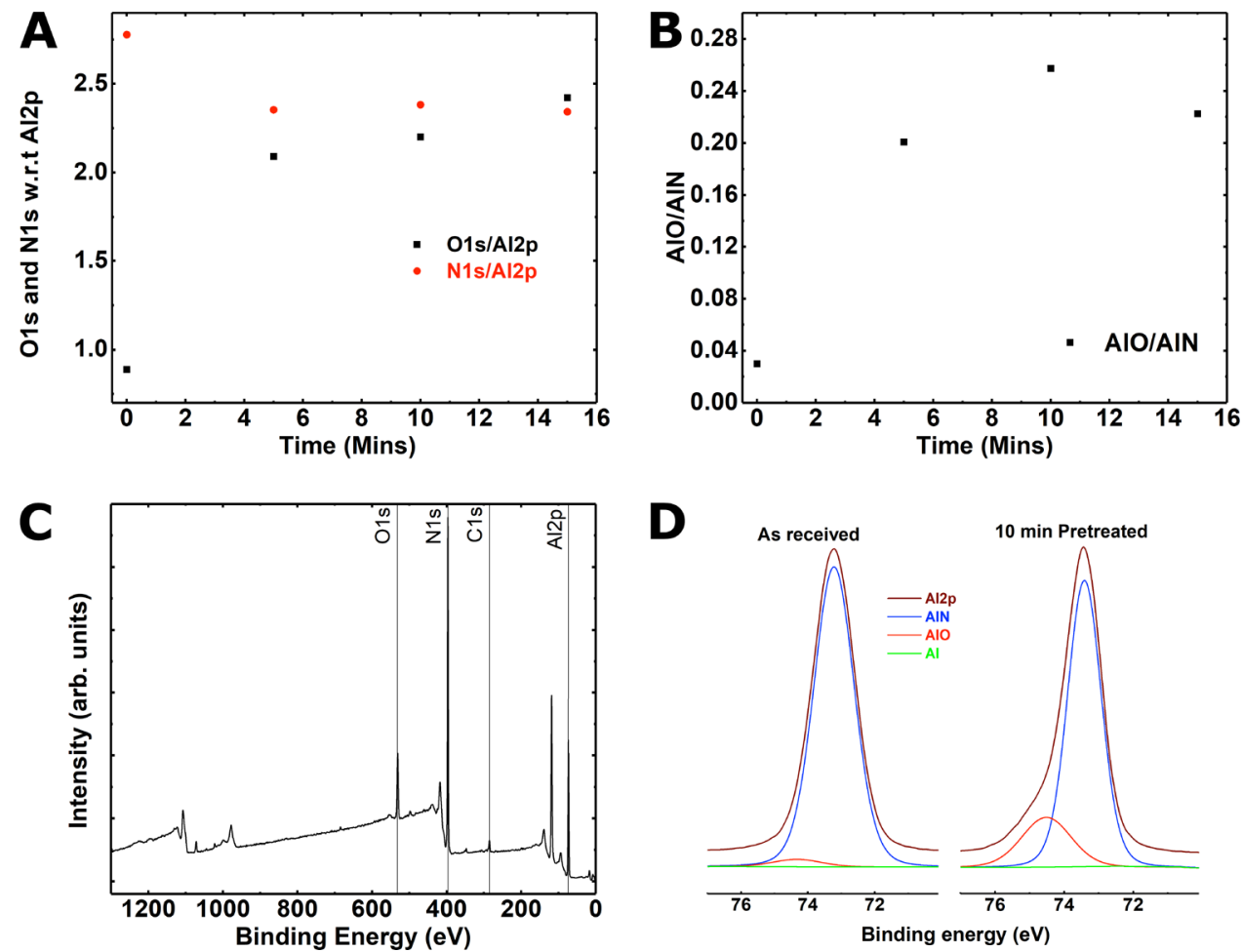

Figure 2. (A) The ratio of $\mathrm{O} 1 \mathrm{~s}$ and $\mathrm{N} 1 \mathrm{~s}$ to $\mathrm{Al} 2 \mathrm{p}$ on the surface of AlN film before and after plasma treatment. (B) The ratio of AlO to AlN peaks in the XPS data. The peaks have been determined by deconvoluting the $\mathrm{Al} 2 \mathrm{p}$ peak in the spectra. (C) A survey spectra for an AlN sample. The vertical lines mark the positions of peaks of interest. (D) $\mathrm{Al} 2 \mathrm{p}$ peak for as-received and 10-min-treated samples. The observed data have been shifted by a fixed amount to show the individual fitted ( $\mathrm{AlN}, \mathrm{AlO}$, and $\mathrm{Al}$ ) peaks.

$\mathrm{H}_{2} / \mathrm{N}_{2}$ plasma, only samples pretreated with this recipe were extensively studied.

The films were then seeded with both $\mathrm{H}$ - and O-terminated seeds. The $\mathrm{H}$-terminated seeds are known to have positive $\zeta$ potential, while the O-terminated ones are negatively charged in water. ${ }^{38}$ Hence, for high seeding density H-terminated or positively charged diamond seeds on the negatively charged AlN layers ${ }^{39}$ need to be used. O-Terminated seeds were also used to cross-check the validity of the measurements. Thick diamond layer was grown on both $\mathrm{O}$ - and $\mathrm{H}$-terminated nanodiamond treated samples. The thick diamond films grown on as-received AlN, treated with both types of seeds, delaminated immediately after growth. Hence, a pretreatment of the AlN layer was devised to increase adhesion. It was found that diamond grown on pretreated samples seeded with $\mathrm{O}$ terminated seeds managed to adhere to the AlN layer. This suggests that lower seeding densities may be useful for making a thick, adherent layer on AlN. The high quality of the diamond grown on the pretreated AlN was demonstrated using Raman spectroscopy and SEM (see Supporting Information).

2.2. Surface Analysis by X-ray Photoelectron Spectroscopy. XPS measurements were performed to study the origin of the $\zeta$-potential previously measured. Figure 2 shows the ratios of various species on the surface of AlN films before and after pretreatment. For the analysis of the spectra, the charge correction was done with respect to the adventitious carbon peak at $285 \mathrm{eV} \cdot{ }^{40,41}$ After the correction, the $\mathrm{O} 1 \mathrm{~s}$ peak was found to be at $531.2 \pm 0.4 \mathrm{eV}$. The data were taken on both as-received as well as samples that were argon-clusteretched in situ to get rid of surface contaminants. In panels A and $\mathrm{B}$ of Figure 2, the data for cluster-etched samples only are shown. In panel $\mathrm{C}$ of the same figure the survey spectra of one of the samples is shown. The vertical lines have been drawn to mark the positions of $\mathrm{Al} \mathrm{2p,C} \mathrm{1s,} \mathrm{N} \mathrm{1s,} \mathrm{and} \mathrm{O} \mathrm{1s} \mathrm{peaks.} \mathrm{The}$ zoomed in $\mathrm{Al} 2 \mathrm{p}$ peaks from the as-received and 10-mintreated samples are shown in Figure 2D. A clear increase in contribution from the $\mathrm{AlO}$ component can be seen in the pretreated sample. The observed spectra have been purposely shifted to show the individual fitted curves.

For deconvoluting the $\mathrm{Al} 2 \mathrm{p}$ peak, three components were chosen, $\mathrm{AlN}, \mathrm{AlO}$, and $\mathrm{Al}$. While none of the samples showed the presence of $\mathrm{Al}$ on the surface (green curve in Figure 2D), the AlN and AlO species had varying concentrations. There is a wide range of values available for such peaks in the literature, ${ }^{42-49}$ the values from Alevli et al. ${ }^{48}$ were taken as our starting point. On the basis of this, certain constraints to the values of $\mathrm{AlO}$ and $\mathrm{Al}$ peaks with respect to the $\mathrm{AlN}$ peak were assigned. The $\mathrm{AlO}$ peak was constrained to be at $+1 \mathrm{eV}$ from the $\mathrm{AlN}$ peak, and the $\mathrm{Al}$ peak was constrained to be at -0.9 $\mathrm{eV}$ from the same peak. All the four samples were analyzed using this method, both before and after cluster etching. The peak position for AlN was found to be $73.3 \pm 0.3 \mathrm{eV}$ and that for $\mathrm{AlO}$ was $74.5 \pm 0.3 \mathrm{eV}$, which is in good agreement with published data.

To see the effects of pretreatment, the total intensities of various peaks in the XPS data were compared. In Figure 2A the ratios of $\mathrm{O} 1 \mathrm{~s}$ and $\mathrm{N} 1 \mathrm{~s}$ peaks to $\mathrm{Al} 2 \mathrm{p}$ peak have been compared. While the $\mathrm{N} 1 \mathrm{~s}$ to $\mathrm{Al} 2 \mathrm{p}$ ratio goes down, the ratio between $\mathrm{O} 1 \mathrm{~s}$ and $\mathrm{Al} 2 \mathrm{p}$ goes up. This is a clear indication that the surface $\mathrm{Al}$ goes from a nitrogen-rich environment to a more oxygen-rich environment. To see the relative concentrations of $\mathrm{AlO}$ and $\mathrm{AlN}$, the two species have been compared in Figure 2B. It is clear that the surface is predominantly nitrogen-rich, but after the treatment, the surface has almost $25 \%$ oxygen. 
This may seem counterintuitive, but the sample is exposed to air between pretreatment and seeding with nano-diamond solution, hence the increase in oxygen content after pretreatment. The increase in the oxygen content at the surface is also validated by a decrease in $\zeta$-potential of AlN films before and after treatment. The ratios of $\mathrm{O} 1 \mathrm{~s}$ and $\mathrm{N} 1$ s to $\mathrm{Al} 2 \mathrm{p}$ for the other treatments have also been measured. The results for those are presented in the Supporting Information. After the pretreatment, the samples were seeded with nano-diamond seeds and were imaged with atomic force microscopy.

2.3. Seeding Density Using Atomic Force Microscopy. From the $\zeta$-potential results, it is clear that $\mathrm{H}$-terminated diamond seeds, which are positively charged in water, should give the highest densities on AlN substrates. The surfaces were seeded with both $\mathrm{H}$ - and O-terminated (negatively charged in water) diamond seeds. AFM was done on seeded and unseeded substrates after plasma pretreatment. The micrographs are shown in Figure 3, where the substrates after
A

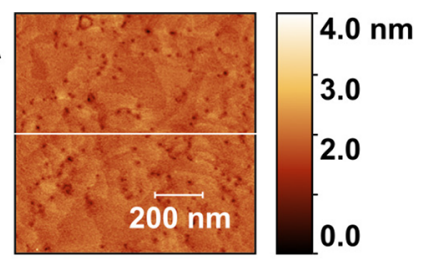

B

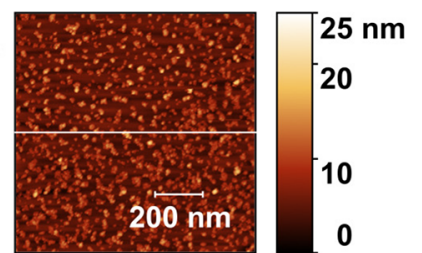

C

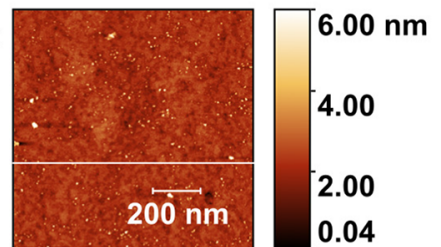

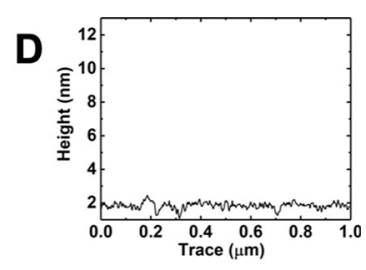

E
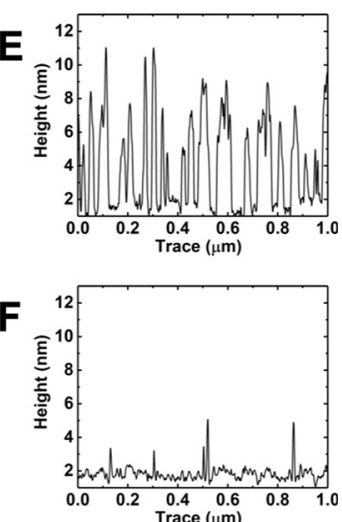

Figure 3. AFM images of AlN wafers before (A) and after seeding with hydrogen (B) and oxygen (C) terminated diamond seed solution. The wafer was plasma pretreated before seeding. Panels D$\mathrm{F}$ are the line profiles of the left column images, as indicated by the white line. The images were taken using a Bruker Dimension Icon Pro in PeakForce Tapping mode.

pretreatment (panel A) and subsequent seeding with different seeds (panels B and C) are shown. Panels D, E, and F are line profiles from images shown in panels $\mathrm{A}, \mathrm{B}$, and $\mathrm{C}$ respectively. The white lines in panels $A, B$, and $C$ show the approximate position of the profile on the image. The AFM images for the untreated substrates with various seedings are shown in the Supporting Information. As expected, high seeding density for $\mathrm{H}$-terminated seeds (panel B) is seen. The line trace in panel $\mathrm{E}$ shows the presence of a large number of seeds on the surface. On the other hand, the line trace in panel F shows very few seeds. However, on comparison with panel D, it can be confirmed that there are some seeds on the surface. The seeding densities observed are in-line with $\zeta$-potential results. The seeded samples were then exposed to growth conditions in a CVD reactor for $30 \mathrm{~s}$, and the images of the uncoalesced films are shown in the Supporting Information. The images clearly show the difference between the $\mathrm{H}$ - and O-treated substrates. The estimated seeding density based on AFM images is $\sim 10^{11} \mathrm{~cm}^{-2}$ for $\mathrm{H}$-terminated diamond seeds and $\sim 10^{9} \mathrm{~cm}^{-2}$ for O-terminated diamond seeds. A number of seeded wafers were used to grow diamond layers of varying thicknesses. Thin diamond layers $(50 \mathrm{~nm})$ were used to study the initial stages of growth by Raman spectroscopy. Layers around $1-\mu \mathrm{m}$-thick were used to study the AlN-diamond interface by TEM and thick diamond layers $(>100 \mu \mathrm{m})$ were finally characterized for their thermal properties.

2.4. Carbon Configuration in Early Growth Stage. Raman measurements were performed in order to investigate the carbon configuration in the early stages of growth. The Raman maps of two 50-nm thin diamond films grown on pretreated and untreated AlN films were taken to understand the diamond-AlN interface. The spectrum was taken over an area of $30 \times 30 \mu \mathrm{m}$. The high-resolution scans were taken between 400 and 1600 and from 1230 to $2300 \mathrm{~cm}^{-1}$. The spectra from two different scans were joined by normalizing the spectra with respect to the $1332 \mathrm{~cm}^{-1}$ diamond peak. ${ }^{50,51}$ The pretreatment in this case was only $10-\mathrm{min}_{2} \mathrm{H}_{2} / \mathrm{N}_{2}$ plasma exposure before seeding. From these maps, representative points were selected, and the Raman data from those points are presented in Figure 4. The red curve is for the sample

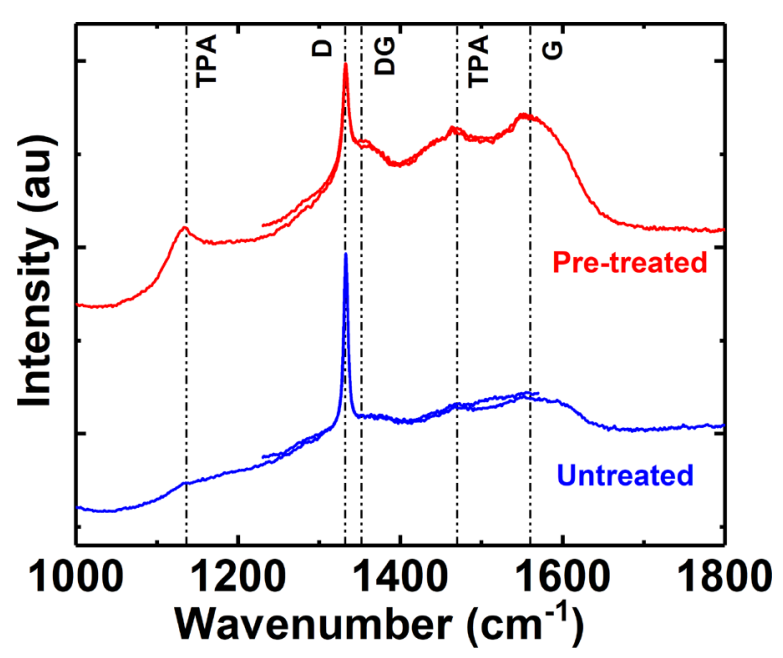

Figure 4. Raman spectroscopy data from very thin diamond films (50 $\mathrm{nm}$ ) grown on pretreated and untreated AlN. The most common peaks have been marked on the figure.

grown on pretreated $\mathrm{AlN}$, and the blue one is for the diamond grown on untreated AlN. Common diamond/non-diamond peak positions of various components are marked in the graph. Spectroscopy data from multiple areas are presented in the Supporting Information to illustrate the homogeneity of the data seen in Figure 4.

The most probable bonding between the diamond and AlN layer is a carbide bond or cyanide bond. The aluminum carbide peaks should appear at 718 and $864 \mathrm{~cm}^{-152}$ and the cyanide peaks should appear as a band around $2090 \mathrm{~cm}^{-1} \cdot{ }^{53,54}$ In all our data, no existence of any peaks or bands is seen. Thus, large-scale bonding through formation of carbide- or cyanidecontaining layers is not detectable by Raman, though this may be related to the presence of only a very thin carbide layer below the Raman detection volume. Looking at the figure, it is evident that there is some significant difference between the non-diamond carbon content in the two thin films. The 
position of trans-polyacetylene (TPA) peaks at 1150 and 1450 $\mathrm{cm}^{-155}$ along with D (DG) and G (G) peaks at 1350 and 1560 $\mathrm{cm}^{-1}, 56$ respectively, have been marked. The disordered carbon peak at $1405 \mathrm{~cm}^{-1}$, linked to graphitic rings, is not present in these thin films, and the shift of the G band from 1580 to 1560 $\mathrm{cm}^{-1}$ is mainly due to switchover of the $\pi$ ring system to $\pi$ chain systems. ${ }^{57}$ All the major peaks related to non-diamond carbon, marked on the Raman spectra, are quite prominent in the film grown on pretreated AlN when compared with film grown on untreated AlN. The large difference in the nondiamond carbon content in the initial phases of growth may be the key to the adhesion of the thick diamond layer on the AlN surface. Raman was also done on thick diamond films on AlN, and the non-diamond peaks are not evident in that spectra (see the Supporting Information). This points to two things: first, the non-diamond carbon is present only during the initial phases of growth, and second, the overall quality of the thick diamond film is good, which is likely to have excellent thermal properties.

2.5. Interface Structure from Cross-Sectional TEM. The atomic and chemical characterization of the AlNdiamond interface was carried out using transmission electron microscopy (TEM). The samples were prepared using a focused ion beam (FIB). An aberration-corrected FEI Titan3 operated at $300 \mathrm{kV}$ was used for high-angle annular dark-field scanning TEM (HAADF-STEM) imaging, and an FEI Tecnai Osiris operated at $200 \mathrm{kV}$ was used for electron energy loss spectroscopy (EELS). The image of the AlN-diamond interface is shown in Figure 5. Voidlike features at the

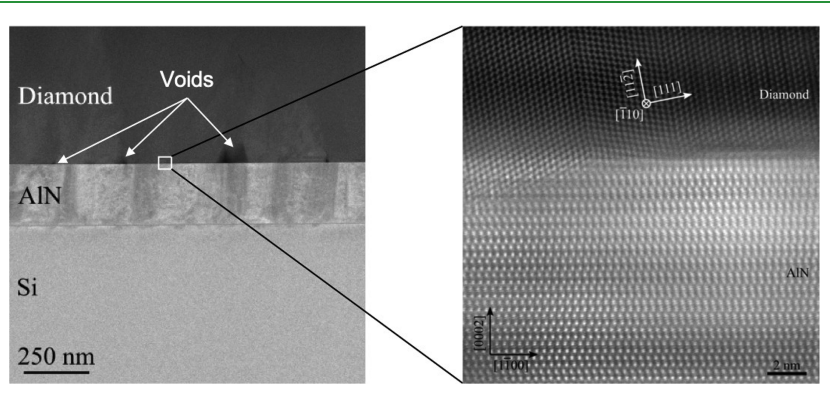

Figure 5. HAAD-STEM image of the sample grown using O-treated diamond seeds. Holes can be seen in places in the diamond film, originating from the seeding. High-resolution TEM images reveal that the interface is sharp with no obvious intermediate phase forming between the AlN and the diamond. It should be noted that the diamond film is polycrystalline and the structure of the interface may vary on the basis of the orientation of the diamond seed relative to the substrate.

interface are clearly visible in the left panel. This is because of the poor seeding density that arises due to the O-terminated seeds used in seeding. The right-hand image shows a clean interface with little clear evidence of any carbide- or cyanidecontaining layers, also absent from the Raman data. This image of the interface is atomically resolved, and that is achieved in this region of the sample because the diamond and AlN lattices are locally well-aligned. This alignment is not, however, typical, since the diamond film is polycrystalline and thus not uniformly aligned to the AlN lattice.

Figure 6 shows the electron energy loss spectroscopy (EELS) analysis of the interface recorded at the same location as the data in Figure 5. The spectra from the bulk of the film and substrate correspond to the standard spectra expected for
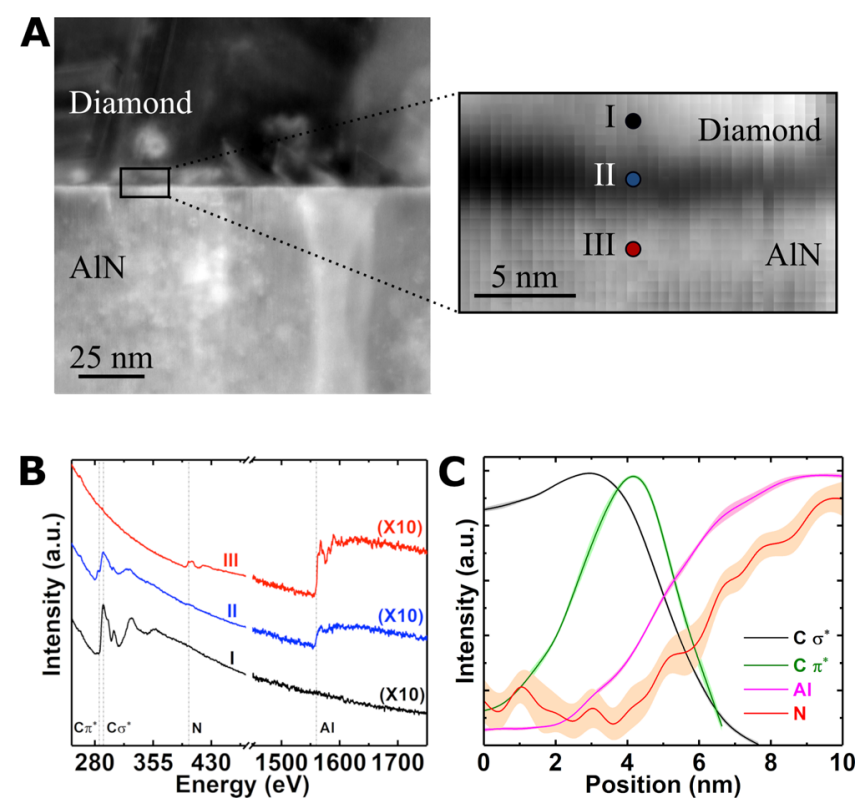

Figure 6. (A) Dark-field image obtained in the same region as the high-resolution image in Figure 5 and the region where the EELS data were recorded. (B) Selected EELS spectra taken of the diamond film (I), at the interface (II), and of the AlN substrate (III); the peaks of interest are labeled. (C) Plot of the (normalized) intensity of the peaks of interest across the interface.

diamond and AlN, respectively. In particular, the C K-edge in the diamond spectrum exhibits only a $\sigma^{*}$ peak, characteristic of the $\mathrm{sp}^{3}$ bonding of diamond. The $\mathrm{C}$ K-edge in the interface region, on the other hand, displays a mixture of $\sigma^{*}$ and $\pi^{*}$ peaks, similar to graphite, which implies $\left(\mathrm{sp}^{2}+2 \mathrm{p}_{z}\right)$ bonding. ${ }^{58}$ It should be noted that the $\mathrm{Al} \mathrm{K}$-edge in the interface region was detected, but no $\mathrm{N}$ K-edge, which may imply the formation of thin carbide right at the interface not detected by Raman. In this specific acquisition, no $\mathrm{O}$ K-edge was detected. Other EELS acquisitions were taken at the interface between the AlN and randomly oriented diamond crystallites. In this instance, the behavior was similar to that reported in Figure 6, apart from an additional O K-edge present at the top surface of the AlN film (but not in the diamond); the presence of $\mathrm{O}$ at the surface of AlN was also suggested from XPS.

2.6. Interfacial and Material Thermal Properties. Transient thermorelfectance measurements were performed to characterize the thermal barrier resistance of the AlNdiamond interface and the thermal conductivity of the diamond films grown on AlN. Figure 7A shows the measured $\mathrm{TBR}_{\text {eff }}$ benchmark of samples studied in this work against the state-of-the art thermal boundary resistance $\left(\mathrm{TBR}_{\text {eff }}\right)$ data of GaN-on-diamond using $\mathrm{Si}_{3} \mathrm{~N}_{4}$ as the interlayer. ${ }^{18}$ We note that the majority of the $\mathrm{GaN}$-on-diamond resistance arises from the $\mathrm{Si}_{3} \mathrm{~N}_{4}$-diamond interface. The experimental details for thermal characterization can be found in the Supporting Information. The most relevant of all the samples is the one with $10-\mathrm{min}$ treatment. The diamond samples grown with the AlN interlayer demonstrate a greatly reduced thermal resistance, with an average barrier resistance of $16 \mathrm{~m}^{2} \cdot \mathrm{K} / \mathrm{GW}$, as shown in Figure 7. This barrier resistance is low in spite of the voids and non-diamond carbon near the interface. The effective thermal conductivity of the AlN interlayer was estimated to be as high as $30 \mathrm{~W} / \mathrm{m} \cdot \mathrm{K}$, by the ratio of AlN thickness $(250 \mathrm{~nm})$ to $\mathrm{TBR}_{\text {eff }}$ this is in contrast to $1-3 \mathrm{~W} / \mathrm{m} \cdot \mathrm{K}^{18}$ for amorphous 

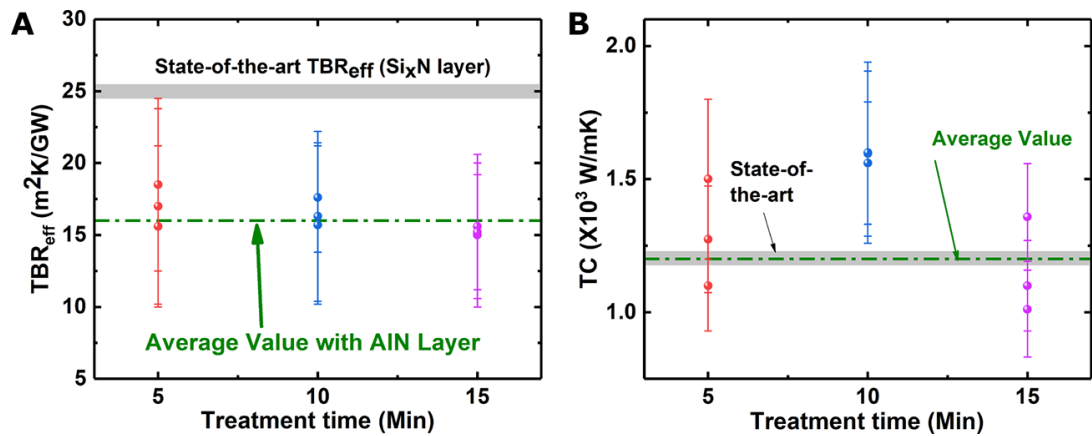

Figure 7. (A) The AlN-diamond effective thermal resistance $\left(\mathrm{TBR}_{\text {eff }}\right)$ measured with the TTR technique. $\mathrm{TBR}_{\text {eff }}$ is a lumped thermal resistance, associated with the thin AlN layer, AlN/diamond boundary, and diamond nucleation layer. Samples were measured with varying pretreatment time. (B) The thermal conductivity of the thick $(\sim 100 \mu \mathrm{m})$ diamond layer grown on the AlN layer.

$\mathrm{Si}_{3} \mathrm{~N}_{4}$ commonly used to seed the diamond growth. This is the reason that the demonstrated diamond-on-AlN approach is so advantageous for diamond-GaN integration. It is to be noted that bulk single-crystalline AlN can have a thermal conductivity as high as $\sim 400 \mathrm{~W} / \mathrm{m} \cdot \mathrm{K}^{59}$ the thermal conductivity of a 250 $\mathrm{nm}$ single-crystalline AlN layer in the sample can be estimated to be about $150 \mathrm{~W} / \mathrm{m} \cdot \mathrm{K}$ using the modified Debye-Callaway model, ${ }^{60}$ suggesting that point defects or grain boundaries reduce its thermal conductivity somewhat. The high-resolution TEM micrograph of the AlN-diamond interface shown in Figure 5 illustrates that there will be only a minor amount of interface roughness phonon scattering at the AlN-diamond interface due to defects. The minimum TBR achievable at the AlN-diamond interface from a diffuse mismatch model (DMM), relying only upon the density of states in these two materials, ${ }^{61}$ is $0.8 \mathrm{~m}^{2} \cdot \mathrm{K} / \mathrm{GW}$.

The average thermal conductivity of the diamond, TC, is $\sim 1200 \mathrm{~W} / \mathrm{m} \cdot \mathrm{K}$ (shown in Figure $7 \mathrm{~B}$ ) for diamond of $\sim 100$ $\mu \mathrm{m}$ thickness. This is comparable to results expected for polycrystalline diamond of similar thickness. ${ }^{14}$ This diamond thermal conductivity measured here is an average thermal conductivity of diamond through the whole layer thickness; with the smaller diamond crystalline size near the AlNdiamond interface, the thermal conductivity of the diamond near this interface is typically reduced. ${ }^{14}$ The high thermal conductivity and sharp $1332 \mathrm{~cm}^{-1}$ peak exhibited in the Raman spectroscopy (see the Supporting Information) demonstrate the high quality of the diamond growth on AlN by this approach. This combined with the low thermal barrier resistance make this approach highly viable for the thermal management of AlN and GaN devices as well as photonics structures.

2.7. Growth Dynamics of Diamond on AIN. A possible model for the adhesion and low thermal barrier resistance demonstrated in this work is shown in Figure 8. During the initial phase of growth (upper panel), the scattered seeds start to grow both vertically and laterally, and there is little to no spontaneous nucleation on areas where there are no diamond seeds, as is also witnessed on silicon. As the nucleation density in this work is significantly below the state-of-the-art (see Figure 3, $\sim 10^{9}$ vs $\sim 10^{11} \mathrm{~cm}^{-2}$ ), the areas that are exposed to the plasma without diamond seeds result in some in-diffusion of carbon, but mostly carbon accumulation on the surface. This carbon is predominantly $\mathrm{sp}^{2}$-bonded, and thus, lower nucleation densities exhibit higher $\mathrm{sp}^{2}$ content at the interface. Eventually, the lateral growth of the diamond crystals blocks these regions from the growth plasma, and small voids are

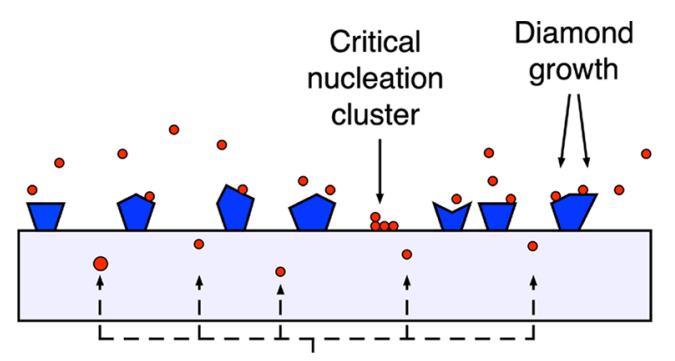

Carbon in-diffusion

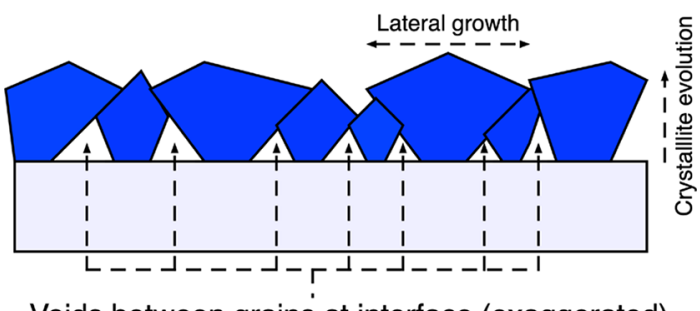

Voids between grains at interface (exaggerated)

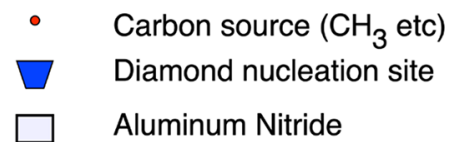

Figure 8. Schematic of diamond film growth with low nucleation density. The initial phase is a mixture of diamond growth on seeds as well as carbon in-diffusion in to the substrate.

enclosed at the interface (lower panel and see Figure 5). These voids may act to reduce stress and promote adhesion in combination with the $\mathrm{sp}^{2}$ bonding. However, these films still exhibit low thermal barrier resistance, and thus, the presence of finite $\mathrm{sp}^{2}$ bonding and voids at the interface is not detrimental to heat flow. This could be due to the lateral growth of the diamond seeds at the interface providing large and abrupt contact between the diamond and AlN, as well as the possible evidence of $\mathrm{Al}-\mathrm{C}$ bonding shown in the EELS (see Figure 6).

\section{CONCLUSIONS}

It is possible to grow a thick $(>100 \mu \mathrm{m})$ diamond layer on AlN. Such a layer could be used for thermal management of $\mathrm{GaN}$ high-power devices. For successful growth, the AlN layers need to be pretreated with $10 \% \mathrm{~N}_{2} / \mathrm{H}_{2}$ plasma for a minimum of $10 \mathrm{~min}$. The pretreatment has been shown to increase the oxygen content of the AlN surface, thus making its $\zeta$-potential more negative. Even though $\mathrm{H}$-treated diamond seeds 
(positively charged seeds on negatively charged surface) seem to be the obvious choice based on $\zeta$-potential study, it was found that films grown after seeding with such seeds delaminated very quickly. As a result, it was found that $\mathrm{O}$ terminated seeds after pretreatment resulted in films that did not delaminate, possibly due to reduced stress at the interface. Considering the fact that thick diamond grown on high-seeddensity substrates delaminated, it is suggested that a low seed density is beneficial for very thick diamond films. Raman spectroscopy studies revealed that the non-diamond carbon content in the initial stages of growth is much higher in films grown on pretreated AlN. It is possible that the excess nondiamond carbon assists in the adhesion of the diamond to AlN layer. The local diamond-AlN interface was found to be extremely abrupt in places. The average thermal barrier resistance measured for the samples grown with O-terminated seeds was found to be $\sim 16 \mathrm{~m}^{2} \cdot \mathrm{K} / \mathrm{GW}$, which is much lower than the current state-of-the-art.

\section{ASSOCIATED CONTENT}

\section{S Supporting Information}

The Supporting Information is available free of charge on the ACS Publications website at DOI: 10.1021/acsami.9b13869.

Details of measurement techniques used, XPS data on $\mathrm{H}_{2}$ - and $\mathrm{O}_{2}$-treated samples, Raman spectra of the thick diamond film, Raman maps of a 50-nm diamond film on AlN, AFM images of seeds on untreated AlN, SEM of a thick, adherent film, and SEM of seeds on AlN after $30 \mathrm{~s}$ of growth (PDF)

\section{AUTHOR INFORMATION}

\section{Corresponding Authors}

*S.M. e-mail: mandals2@cardiff.ac.uk, soumen.mandal@gmail. com.

*M.K. e-mail: martin.kuball@bristol.ac.uk.

*O.A.W. e-mail: williamso@cardiff.ac.uk.

\section{ORCID $\odot$}

Soumen Mandal: 0000-0001-8912-1439

Fabien Massabuau: 0000-0003-1008-1652

Jerome Cuenca: 0000-0003-1370-1167

Tim Batten: 0000-0002-3245-4985

David Morgan: 0000-0002-6571-5731

\section{Notes}

The authors declare no competing financial interest.

Dataset. The datasets generated and/or analysed during this study can be found at http://doi.org/10.17035/ d.2019.0079285250.

\section{ACKNOWLEDGMENTS}

The authors would like to acknowledge financial support of the Engineering and Physical Sciences Research Council under the program Grant GaN-DaME (EP/P00945X/1). S.M. and O.A.W. would like to acknowledge financial support from the European Research Council under the EU Consolidator Grant SUPERNEMS. D.J.W. would like to acknowledge financial support of the Engineering and Physical Sciences Research Council under the EPSRC Fellowship grant (EP/ N01202X/2). S.M. would like to thank Dr Andreas Papageorgiou for help with Python codes for analyzing the Raman data. We would like to thank Manikant (Bristol) for preparing a cross-sectional view of the thick sample.

\section{REFERENCES}

(1) Mishra, U.; Parikh, P.; Wu, Y.-F AlGaN/GaN HEMTs-An Overview of Device Operation and Applications. Proc. IEEE 2002, 90, 1022-1031.

(2) Mishra, U.; Shen, L.; Kazior, T.; Wu, Y.-F. GaN-Based RF Power Devices and Amplifiers. Proc. IEEE 2008, 96, 287-305.

(3) Sangmin, L.; Vetury, R.; Brown, J. D.; Gibb, S. R.; Cai, W. Z.; Jinming, S.; Green, D. S.; Shealy, J. Reliability Assessment of AlGaN/ GaN HEMT Technology on SiC for 48V Applications. 2008 IEEE International Reliability Physics Symposium; IEEE, 2008; , pp 446-449.

(4) Pomeroy, J. W.; Uren, M. J.; Lambert, B.; Kuball, M. Operating Channel Temperature in GaN HEMTs: DC versus RF Accelerated Life Testing. Microelectron. Reliab. 2015, 55, 2505-2510.

(5) Gaska, R.; Osinsky, A.; Yang, J. W.; Shur, M. S. Self-Heating in High-Power AlGaN-GaN HFET's. IEEE Electron Device Lett. 1998, 19, 89-91.

(6) Kuball, M.; Hayes, J. M.; Uren, M. J.; Martin, T.; Birbeck, J. C. H.; Balmer, R. S.; Hughes, B. T. Measurement of Temperature in Active High-Power AlGaN/GaN HFETs Using Raman Spectroscopy. IEEE Electron Device Lett. 2002, 23, 7-9.

(7) Goldberg, Y.; Levinshtein, M.; Rumyantsev, S. In Properties of Advanced SemiconductorMaterials GaN, AlN, SiC, BN, SiC, SiGe; Levinshtein, M., Rumyantsev, S., Shur, M., Eds.; John Wiley \& Sons, Inc.: New York, 2001; pp 93-148.

(8) Ho, C. Y.; Powell, R. W.; Liley, P. E. Thermal Conductivity of the Elements. J. Phys. Chem. Ref. Data 1972, 1, 279-421.

(9) Coe, S.; Sussmann, R. Optical, Thermal and Mechanical Properties of CVD Diamond. Diamond Relat. Mater. 2000, 9, 17261729.

(10) Ivakin, E. V.; Sukhodolov, A. V.; Ralchenko, V. G.; Vlasov, A. V.; Khomich, A. V. Measurement of Thermal Conductivity of Polycrystalline CVD Diamond by Laser-Induced Transient Grating Technique. Quantum Electron. 2002, 32, 367-372.

(11) Pomeroy, J. W.; Bernardoni, M.; Dumka, D. C.; Fanning, D. M.; Kuball, M. Low Thermal Resistance GaN-on-Diamond Transistors Characterized by Three-Dimensional Raman Thermography Mapping. Appl. Phys. Lett. 2014, 104, 083513.

(12) Schreck, M.; Asmussen, J.; Shikata, S.; Arnault, J.-C.; Fujimori, N. Large-Area High-Quality Single Crystal Diamond. MRS Bull. 2014, 39, 504-510.

(13) Schreck, M.; Gsell, S.; Brescia, R.; Fischer, M. Ion Bombardment Induced Buried Lateral Growth: the Key Mechanism for the Synthesis of Single Crystal Diamond Wafers. Sci. Rep. 2017, 7, 44462.

(14) Anaya, J.; Sun, H.; Pomeroy, J.; Kuball, M. Thermal Management of GaN-on-Diamond High Electron Mobility Transistors: Effect of the Nanostructure in the Diamond Near Nucleation Region. 2016 15th IEEE Intersociety Conference on Thermal and Thermomechanical Phenomena in Electronic Systems (ITherm); IEEE, 2016; , pp 1558-1565.

(15) Jessen, G. H.; Gillespie, J. K.; Via, G. D.; Crespo, A.; Langley, D.; Wasserbauer, J.; Faili, F.; Francis, D.; Babic, D.; Ejeckam, F.; Guo, S.; Eliashevich, I. AlGaN/GaN HEMT on Diamond Technology Demonstration. 2006 IEEE Compound Semiconductor Integrated Circuit Symposium; IEEE, 2006; , pp 271-274.

(16) Hirama, K.; Taniyasu, Y.; Kasu, M. AlGaN/GaN High-Electron Mobility Transistors with Low Thermal Resistance Grown on SingleCrystal Diamond (111) Substrates by Metalorganic Vapor-Phase Epitaxy. Appl. Phys. Lett. 2011, 98, 162112.

(17) Dumka, D. C.; Chou, T. M.; Jimenez, J. L.; Fanning, D. M.; Francis, D.; Faili, F.; Ejeckam, F.; Bernardoni, M.; Pomeroy, J. W.; Kuball, M. Electrical and Thermal Performance of AlGaN/GaN HEMTs on Diamond Substrate for RF Applications. 2013 IEEE Compound Semiconductor Integrated Circuit Symposium (CSICS); IEEE, 2013; , pp 1-4.

(18) Sun, H.; Simon, R. B.; Pomeroy, J. W.; Francis, D.; Faili, F.; Twitchen, D. J.; Kuball, M. Reducing GaN-on-Diamond Interfacial Thermal Resistance for High Power Transistor Applications. Appl. Phys. Lett. 2015, 106, 111906. 
(19) Mandal, S.; Thomas, E. L. H.; Middleton, C.; Gines, L.; Griffiths, J. T.; Kappers, M. J.; Oliver, R. A.; Wallis, D. J.; Goff, L. E.; Lynch, S. A.; Kuball, M.; Williams, O. A. Surface Zeta Potential and Diamond Seeding on Gallium Nitride Films. ACS Omega 2017, 2, $7275-7280$.

(20) May, P.; Rego, C.; Trevor, C.; Williamson, E.; Ashfold, M.; Rosser, K.; Everitt, N. Deposition of Diamond Films on Sapphire: Studies of Interfacial Properties and Patterning Techniques. Diamond Relat. Mater. 1994, 3, 1375-1380.

(21) Zhu, D.; Wallis, D. J.; Humphreys, C. J. Prospects of III-Nitride Optoelectronics Grown on Si. Rep. Prog. Phys. 2013, 76, 106501.

(22) Kuball, M.; Pomeroy, J. W.; Uren, M.; Williams, O. A. A Semiconductor on Diamond Substrate, Precursor for Use in Preparing a Semiconductor on Diamond Substrate, and Methods for Making the Same. GB Patent GB1814192.9, 2019.

(23) Pomeroy, J.; Bernardoni, M.; Sarua, A.; Manoi, A.; Dumka, D. C.; Fanning, D. M.; Kuball, M. Achieving the Best Thermal Performance for GaN-on-Diamond. 2013 IEEE Compound Semiconductor Integrated Circuit Symposium (CSICS); IEEE, 2013; , pp 14.

(24) Godbole, V. P.; Jagannadham, K.; Narayan, J. Nucleation and Growth of Diamond Films on Aluminum Nitride Coated Nickel. Appl. Phys. Lett. 1995, 67, 1322.

(25) Cui, J.; Ma, Y.; Zhang, J.; Chen, H.; Fang, R. Growth and Characterization of Diamond Film on Aluminum Nitride. Mater. Res. Bull. 1996, 31, 781-785.

(26) Wang, W. L.; Zhang, R. Q.; Liao, K. J.; Sun, Y. W.; Wang, B. B. Nucleation and Growth of Diamond films on Aluminum Nitride by Hot Filament Chemical Vapor Deposition. Diamond Relat. Mater. 2000, 9, 1660-1663.

(27) Hees, J.; Heidrich, N.; Pletschen, W.; Sah, R. E.; Wolfer, M.; Williams, O. A.; Lebedev, V.; Nebel, C. E.; Ambacher, O. Piezoelectric Actuated Micro-Resonators Based on the Growth of Diamond on Aluminum Nitride Thin Films. Nanotechnology 2013, 24, 025601.

(28) Cervenka, J.; Dontschuk, N.; Ladouceur, F.; Duvall, S. G.; Prawer, S. Diamond/Aluminium Nitride Composites for Efficient Thermal Management Applications. Appl. Phys. Lett. 2012, 101, 051902.

(29) Pobedinskas, P.; Degutis, G.; Dexters, W.; Hardy, A.; Van Bael, M. K.; Haenen, K. Influence of Hydrogen and Hydrogen/Methane Plasmas on AlN Thin Films. Appl. Phys. Lett. 2014, 104, 081917.

(30) Cervenka, J.; Lau, D. W. M.; Dontschuk, N.; Shimoni, O.; Silvestri, L.; Ladouceur, F.; Duvall, S. G.; Prawer, S. Nucleation and Chemical Vapor Deposition Growth of Polycrystalline Diamond on Aluminum Nitride: Role of Surface Termination and Polarity. Cryst. Growth Des. 2013, 13, 3490-3497.

(31) Pobedinskas, P.; Degutis, G.; Dexters, W.; Janssen, W.; Janssens, S. D.; Conings, B.; Ruttens, B.; D’Haen, J.; Boyen, H.-G.; Hardy, A.; Van Bael, M. K.; Haenen, K. Surface Plasma Pretreatment for Enhanced Diamond Nucleation on AlN. Appl. Phys. Lett. 2013, 102, 201609.

(32) Philip, J.; Hess, P.; Feygelson, T.; Butler, J. E.; Chattopadhyay, S.; Chen, K. H.; Chen, L. C. Elastic, Mechanical, and Thermal Properties of Nanocrystalline Diamond Films. J. Appl. Phys. 2003, 93, 2164-2171.

(33) Sumant, A. V.; Gilbert, P.; Grierson, D. S.; Konicek, A. R.; Abrecht, M.; Butler, J. E.; Feygelson, T.; Rotter, S. S.; Carpick, R. W. Surface Composition, Bonding, and Morphology in the Nucleation and Growth of Ultra-Thin, High Quality Nanocrystalline Diamond Films. Diamond Relat. Mater. 2007, 16, 718-724.

(34) Rotter, S. Z.; Madaleno, J. C. Diamond CVD by a Combined Plasma Pretreatment and Seeding Procedure. Chem. Vap. Deposition 2009, 15, 209-216.

(35) Lee, H.-J.; Jeon, H.; Lee, W.-S. Ultrathin Ultrananocrystalline Diamond Film Synthesis by Direct Current Plasma-Assisted Chemical Vapor Deposition. J. Appl. Phys. 2011, 110, 084305.

(36) Muchnikov, A. B.; Vikharev, A. L.; Butler, J. E.; Chernov, V. V.; Isaev, V. A.; Bogdanov, S. A.; Okhapkin, A. I.; Yunin, P. A.; Drozdov,
Y. N. Homoepitaxial Growth of CVD Diamond After ICP Pretreatment. Phys. Status Solidi A 2015, 212, 2572-2577.

(37) Bland, H. A.; Thomas, E. L. H.; Klemencic, G. M.; Mandal, S.; Morgan, D. J.; Papageorgiou, A.; Jones, T. G.; Williams, O. A. Superconducting Diamond on Silicon Nitride for Device Applications. Sci. Rep. 2019, 9, 2911.

(38) Hees, J.; Kriele, A.; Williams, O. A. Electrostatic Self-Assembly of Diamond Nanoparticles. Chem. Phys. Lett. 2011, 509, 12-15.

(39) Williams, O. A.; Douhéret, O.; Daenen, M.; Haenen, K.; Ōsawa, E.; Takahashi, M. Enhanced Diamond Nucleation on Monodispersed Nanocrystalline Diamond. Chem. Phys. Lett. 2007, $445,255-258$.

(40) Wilson, J.; Walton, J.; Beamson, G. Analysis of Chemical Vapour Deposited Diamond Films by X-ray Photoelectron Spectroscopy. J. Electron Spectrosc. Relat. Phenom. 2001, 121, 183-201.

(41) Ferro, S.; Dal Colle, M.; De Battisti, A. Chemical Surface Characterization of Electrochemically and Thermally Oxidized Borondoped Diamond Film Electrodes. Carbon 2005, 43, 1191-1203.

(42) Youngman, R. A.; Harris, J. H. Luminescence Studies of Oxygen-Related Defects In Aluminum Nitride. J. Am. Ceram. Soc. 1990, 73, 3238-3246.

(43) Liao, H. M.; Sodhi, R. N. S.; Coyle, T. W. Surface Composition of AlN Powders Studied by X-ray Photoelectron Spectroscopy and Bremsstrahlung Excited Auger Electron Spectroscopy. J. Vac. Sci. Technol., A 1993, 11, 2681-2686.

(44) Kazan, M.; Rufflé, B.; Zgheib, C.; Masri, P. Oxygen Behavior in Aluminum Nitride. J. Appl. Phys. 2005, 98, 103529.

(45) Kim, O. H.; Kim, D.; Anderson, T. Atomic Layer Deposition of GaN Using $\mathrm{GaCl}_{3}$ and $\mathrm{NH}_{3}$. J. Vac. Sci. Technol., A 2009, 27, 923928.

(46) Jose, F.; Ramaseshan, R.; Dash, S.; Bera, S.; Tyagi, A. K.; Raj, B. Response of Magnetron Sputtered AlN Films to Controlled Atmosphere Annealing. J. Phys. D: Appl. Phys. 2010, 43, 075304.

(47) García-Méndez, M.; Morales-Rodríguez, S.; Shaji, S.; Krishnan, B.; Bartolo-Pérez, P. Structural Properties of AlN Films with Oxygen Content Deposited by Reactive Magnetron Sputtering: XRD and XPS Characterization. Surf. Rev. Lett. 2011, 18, 23-31.

(48) Alevli, M.; Ozgit, C.; Donmez, I.; Biyikli, N. Structural Properties of AlN Films Deposited by Plasma-enhanced Atomic Layer Deposition at Different Growth Temperatures. Phys. Status Solidi A 2012, 209, 266-271.

(49) Motamedi, P.; Cadien, K. XPS Analysis of AlN Thin Films Deposited by Plasma Enhanced Atomic Layer Deposition. Appl. Surf. Sci. 2014, 315, 104-109.

(50) Ramaswamy, C. Raman Effect in Diamond. Nature 1930, 125, 704.

(51) Bhagavantam, S. Relation of Raman Effect to Crystal Structure. Indian J. Phys. 1930, 5, 169.

(52) Kennedy, J. L.; Drysdale, T. D.; Gregory, D. H. Rapid, EnergyEfficient Synthesis of the Layered Carbide, $\mathrm{Al}_{4} \mathrm{C}_{3}$. Green Chem. 2015, $17,285-290$

(53) Seki, H.; Samant, M. G. Use of Cassegrain System for in Situ Raman Scattering Study of Cyanide Ions on a Palladium Electrode. Appl. Spectrosc. 1993, 47, 952-956.

(54) Cosano, D.; Esquinas, C.; Jiménez-Sanchidrián, C.; Ruiz, J. R. Use of Raman Spectroscopy to Assess the Efficiency of MgAl Mixed Oxides in Removing Cyanide from Aqueous Solutions. Appl. Surf. Sci. 2016, 364, 428-433.

(55) Ferrari, A. C.; Robertson, J. Origin of the $1150 \mathrm{~cm}^{-1}$ Raman Mode in Nanocrystalline Diamond. Phys. Rev. B: Condens. Matter Mater. Phys. 2001, 63, 121405.

(56) May, P.; Smith, J.; Rosser, K. 785 nm Raman Spectroscopy of CVD Diamond Films. Diamond Relat. Mater. 2008, 17, 199-203.

(57) Ferrari, A. C.; Robertson, J. Raman Spectroscopy of Amorphous, Nanostructured, Diamond Like Carbon, and Nanodiamond. Philos. Trans. R. Soc., A 2004, 362, 2477-2512.

(58) Hamon, A.-L.; Verbeeck, J.; Schryvers, D.; Benedikt, J.; M. C. M. v. d. Sanden, R. ELNES Study of Carbon K-edge Spectra of Plasma Deposited Carbon Films. J. Mater. Chem. 2004, 14, 2030. 
(59) Rounds, R.; Sarkar, B.; Klump, A.; Hartmann, C.; Nagashima, T.; Kirste, R.; Franke, A.; Bickermann, M.; Kumagai, Y.; Sitar, Z.; Collazo, R. Thermal Conductivity of Single-Crystalline AlN. Appl. Phys. Express 2018, 11, 071001.

(60) Morelli, D. T.; Heremans, J. P.; Slack, G. A. Estimation of the Isotope Effect on the Lattice Thermal Conductivity of Group IV and Group III-V Semiconductors. Phys. Rev. B: Condens. Matter Mater. Phys. 2002, 66, 195304.

(61) Swartz, E. T.; Pohl, R. O. Thermal Boundary Resistance. Rev. Mod. Phys. 1989, 61, 605-668. 